\title{
Identification of potential genes and miRNAs associated with sepsis based on microarray analysis
}

\author{
YIN LI* , FENGXIA ZHANG ${ }^{*}$, YAN CONG and YUN ZHAO \\ Emergency Department, Huadong Hospital, Shanghai 200040, P.R. China
}

Received May 10, 2017; Accepted September 18, 2017

DOI: $10.3892 / \mathrm{mmr} .2018 .8668$

\begin{abstract}
Sepsis is a type of systemic inflammatory response syndrome caused by infection. The present study aimed to examine key genes and microRNAs (miRNAs) involved in the pathogenesis of sepsis. The GSE13205 microarray dataset, downloaded from the Gene Expression Omnibus was analyzed using bioinformatics tools, and included muscle biopsy specimens of 13 patients with sepsis and eight healthy controls. The differentially expressed genes (DEGs) in samples from patients with sepsis were identified using the Linear Models for Microarray package in R language. Using the Database for Annotation, Visualization and Integration Discovery tool, functional and pathway enrichment analyses were performed to examine the potential functions of the DEGs. The protein-protein interaction (PPI) network was constructed with the DEGs using the Search Tool for the Retrieval of Interacting Genes, and the network topology was analyzed using CytoNCA. Subsequently, MCODE in Cytoscape was used to identify modules in the PPI network. Finally, the integrated regulatory network was constructed based on the DEGs, miRNAs and transcription factors (TFs). A total of 259 upregulated DEGs (MYC and BYSL) and 204 downregulated DEGs were identified in the patients with sepsis. NOP14, NOP2, AATF, GTPBP4, BYSL and TRMT6 were key genes in
\end{abstract}

Correspondence to: Mr. Yun Zhao, Emergency Department, Huadong Hospital, 221 Yan'an Xi Road, Shanghai 200040, P.R. China

E-mail: zhaoyun1155@163.com

*Contributed equally

Abbreviations: miRNA, microRNA; DEGs, differentially expressed genes; DAVID, database for annotation, visualization and integration discovery; GEO, gene expression omnibus; PPI, protein-protein interaction; LIMMA, linear models for microarray; TFs, transcription factors; SIRS, systemic inflammatory response syndrome; IL-6, interleukin-6; TNF- $\alpha$, tumor necrosis factor- $\alpha$; LPS, lipopolysaccharides; GO, gene ontology; KEGG, Kyoto Encyclopedia of Genes and Genomes; FC, fold change

Key words: sepsis, microRNA, target gene, protein-protein interaction, integrated regulatory network the MCODE module. In the integrated DEG-miRNA-TF regulatory network, hsa-miR-150 (target gene MYLK3) and 21 TFs, comprising 14 upregulated DEGs (including $M Y C$ ) and seven downregulated DEGs, were identified. The results suggested that NOP14, NOP2, AATF, GTPBP4, BYSL, MYC, MYLK3 and miR-150 may be involved in the pathogenesis of sepsis.

\section{Introduction}

Sepsis, a type of systemic inflammatory response syndrome (SIRS), is a complication caused by the response of the whole body, and injured tissues and organs to an infection (1). In the last decade, the incidence rates of sepsis and severe sepsis in the population were 4.37 and 2.70 cases per 1,000 individuals, respectively, in high-income-countries (including seven countries on four continents); annually, the global mortality rate is as high as 5,300,000 (2). In China, a survey revealed that, 10 years ago, the incidence and mortality rates of sepsis were 8.68 and $48.7 \%$, respectively (3). It is difficult to predict, diagnose and treat patients with sepsis (4). In addition, there are no effective drugs or medical interventions for treating this disease. Therefore, the investigation of target genes and microRNAs (miRNAs) in sepsis is urgently required.

Proinflammatory cytokines, including interleukin-6 (IL-6) and tumor necrosis factor- $\alpha$ (TNF- $\alpha$ ), have been implicated as key mediators in inflammation associated with sepsis (5). Nrf2, a basic leucine zipper transcription factor, is a regulatory factor for response to lipopolysaccharides (LPS) and TNF- $\alpha$ by activating the expression of nuclear factor $-\kappa \mathrm{B}(\mathrm{NF}-\kappa \mathrm{B})$ during experimental sepsis (6). PTP1B is a target gene for the treatment of sepsis via the reduction of cardiovascular inflammation (7). In addition, miRNAs are small non-coding RNA molecules of 20-23 nucleotides present in almost all living things, ranging from viruses to higher animals (8). miRNAs have various biological functions and are involved in inflammation, metabolism and tumor processes through a series of signaling pathways (9). miRNA (miR)-34a (10), miR-150 (11) and miR-15a (10) are considered to be novel indicators for the early diagnosis and prognosis of sepsis. However, the role of target genes and miRNAs in sepsis requires further elucidation. To clarify the role of key genes and miRNAs in sepsis, the present study aimed to identify potential genes and miRNAs associated with sepsis, based on the GSE13205 dataset from the Gene Expression Omnibus (GEO) database (12). 
The present study identified differentially expressed genes (DEGs) in sepsis, and then analyzed the potential functions of these DEGs using Gene Ontology (GO) functional and Kyoto Encyclopedia of Genes and Genomes (KEGG) pathway enrichment analyses. Subsequently, a protein-protein interaction (PPI) network between the DEGs was constructed and GO-associated analysis of the PPI network module was performed. Finally, an integrated regulatory network was constructed based on the DEGs, miRNAs and transcription factors (TFs) for the prediction of key targets in sepsis.

\section{Materials and methods}

Microarray data. The gene expression profile of GSE13205 was downloaded from the GEO database (http://www.ncbi. nlm.nih.gov/geo/), which included muscle biopsy specimens from 13 patients with sepsis from the General Intensive Care Unit at Karolinska University Hospital (Huddinge, Sweden) and eight healthy controls from Ersta Hospital (Stockholm, Sweden). Muscle biopsy samples were obtained from the lateral portion of the vastus lateralis muscle $(10-20 \mathrm{~cm}$ above the knee). The GSE13205 profile was deposited by Fredriksson et al according to the platform of GPL570 [HG-U133_Plus_2] Affymetrix Human Genome U133 Plus 2.0 Array. Their study was approved by the Ethical Committee of Karolinska Institutet (Stockholm, Sweden) and received local ethics approval for gene expression analysis at Heriot-Watt University (Edinburgh, Scotland) (13). Informed consent was obtained from all patients or close relatives.

Data preprocessing and identification of DEGs. The microarray data were normalized using the robust multi-array average function in the Affy package (version 1.52.0; http://bioconductor.org/packages/release/bioc/html/affy.html)of $\mathrm{R}$ software (version 3.3.2; https://cran.r-project. org/bin/windows/base/) (14). Subsequently, the Bayesian method of the Linear Models for Microarray (LIMMA; version 3.30.3; http://bioconductor.org/packages/release/bioc/html/limma.html) package in $\mathrm{R}$ was used for identifying the DEGs between the samples from patients with sepsis and those from the healthy controls (15). The threshold value of DEGs was set as llog fold change (FC) $\mid>1$ and $\mathrm{P}<0.05$.

GO and KEGG pathway enrichment analyses. GO analysis (http://geneontology.org/page/go-enrichment-analysis) has become a predictable method for enrichment analysis to understand biological process (BP), cellular localization and molecular function (16). KEGG (http://www.genome. $\mathrm{jp} / \mathrm{kegg} /$ ) is a well-known database for the systematic analysis of gene function and genomic information, including the GENES and PATHWAY databases (17). The Database for Annotation, Visualization and Integration Discovery (DAVID; version 6.7; https://david-d.ncifcrf.gov/) is a public high-throughput functional annotation tool, which provides functional annotation bioinformatics microarray analysis for integrating data-mining environments and analyzing gene lists (18). The DEGs were input into DAVID online for GO and KEGG enrichment analyses. $\mathrm{P}<0.05$ was considered to be statistically significant.

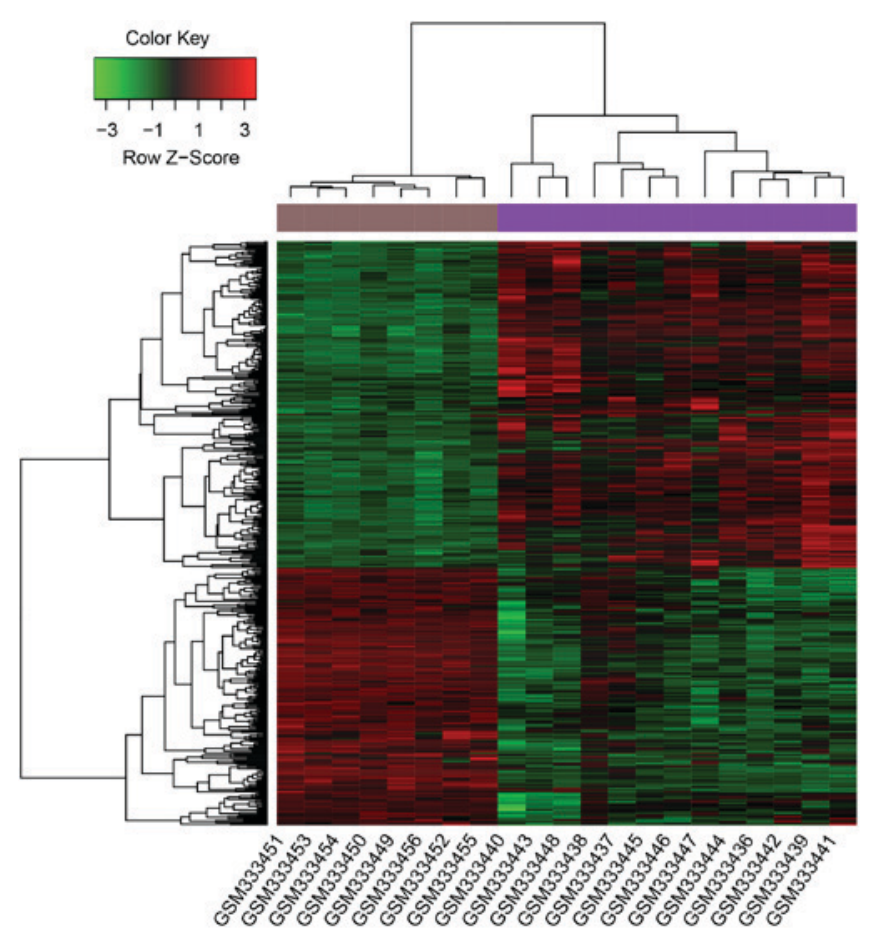

Figure 1. Heatmap of clustering analysis. The heatmap showed all significant DEGs ( $\mid \log$ fold changel $>1$ and $\mathrm{P}<0.05$ ) identified from the comparison of samples from patients with sepsis, vs. those from healthy controls. GSM333436-GSM333448 (purple) indicates the sepsis group and GSM333449-GSM333456 (brown) indicates the healthy control group. The red color indicates upregulated DEGs and the green color indicates downregulated DEGs, compared with corresponding samples from healthy controls; DEGs, differentially expressed genes.

Analysis of the PPI network. The PPI network was constructed with all the DEGs using the Search Tool for the Retrieval of Interacting Genes (STRING) from a well-known online server (version 10.0; http://www.string-db.org/) (19). PPI links with a combined score $>0.4$ were identified for constructing the PPI network. The PPI network was visualized using Cytoscape software (http://cytoscape.org/), and the network topology was analyzed using CytoNCA (version 2.1.6; http://apps.cytoscape. org/apps/cytonca) (20). The parameter was set as 'without weight'.

GO-associated analysis of the PPI network module. MCODE in Cytoscape was used to search for modules in the PPI network (21). The modules were identified when the degree of cut-off was 2 , the node score cut-off was 0.2 , the K-core was 2 , and the maximum depth was 100 . GO-associated analysis was performed on the module with the maximum score using Golorize (version: 1.0.0.beta1) (22). In addition, the top five GO terms in the module were analyzed.

Construction of the integrated regulatory network. The integrated regulatory network was constructed based on the DEGs, miRNAs and TFs. miRNAs associated with DEGs were identified from the mir2disease database (http://www.mir2disease.org/) and miRWalk (http://zmf. umm.uni-heidelberg.de/apps/zmf/mirwalk2/) $(23,24)$. Finally, the miRNA-target gene links, which overlapped with DEGs, were considered to be closely associated with sepsis. TFs were selected from the integrated transcription factor platform 
Table I. Results of GO and KEGG enrichment on upregulated differentially expressed genes.

\begin{tabular}{|c|c|c|c|}
\hline Category & Term & Count & P-value \\
\hline GO term BP & GO:0045768-Positive regulation of anti-apoptosis & 5 & $6.2 \mathrm{E}-4$ \\
\hline GO term BP & GO:0045767-Regulation of anti-apoptosis & 5 & $1.5 \mathrm{E}-3$ \\
\hline GO term BP & GO:0022613-Ribonucleoprotein complex biogenesis & 9 & 2.7E-3 \\
\hline GO term BP & GO:0010033-Response to organic substance & 20 & $3.2 \mathrm{E}-3$ \\
\hline GO term BP & GO:0006366-Transcription from RNA polymerase II promoter & 10 & $3.9 \mathrm{E}-3$ \\
\hline KEGG pathway & Hsa04210: Apoptosis & 5 & $1.3 \mathrm{E}-2$ \\
\hline KEGG pathway & Hsa00480: Glutathione metabolism & 4 & $1.6 \mathrm{E}-2$ \\
\hline
\end{tabular}

GO, gene ontology; KEGG, Kyoto Encyclopedia of Genes and Genomes; BP, biological process.

Table II. Results of GO and KEGG enrichment on downregulated differentially expressed genes.

\begin{tabular}{llrr}
\hline Category & \multicolumn{1}{c}{ Term } & Count & P-value \\
\hline GO term BP & GO:0007517-Muscle organ development & 14 & $3.68 \mathrm{E}-8$ \\
GO term BP & GO:0006936-Muscle contraction & 11 & $8.74 \mathrm{E}-7$ \\
GO term BP & GO:0003012-Muscle system process & 11 & $2.05 \mathrm{E}-6$ \\
GO term BP & GO:0044057-Regulation of system process & 13 & $1.60 \mathrm{E}-5$ \\
GO term BP & GO:0006942-Regulation of striated muscle contraction & 5 & $3.56 \mathrm{E}-5$ \\
KEGG pathway & Hsa04020: Calcium signaling pathway & $1.40 \mathrm{E}-3$ \\
KEGG pathway & Hsa04960: Aldosterone-regulated sodium reabsorption & $4.30 \mathrm{E}-3$ \\
KEGG pathway & Hsa04270: Vascular smooth muscle contraction & 5 & $2.36 \mathrm{E}-2$ \\
KEGG pathway & Hsa04920: Adipocytokine signaling pathway & 4 & $2.75 \mathrm{E}-2$ \\
KEGG pathway & Hsa04260: Cardiac muscle contraction & 4 & $4.06 \mathrm{E}-2$ \\
\hline
\end{tabular}

GO, gene ontology; KEGG, Kyoto Encyclopedia of Genes and Genomes; BP, biological process.

(http://itfp.biosino.org/itfp) (25) and TRANSFAC (http://www. gene-regulation.com/pub/databases.html) among DEGs (26). The DEG-miRNA-TF network was constructed and visualized using Cytoscape software.

\section{Results}

DEGs in sepsis. A total of 463 DEGs were identified from patients with sepsis and healthy controls, which comprised 259 upregulated DEGs, including $M Y C$ and BYSL, and 204 downregulated DEGs, including MYLK3. The clustering heatmap showed that DEGs were well distinguished between samples from patients with sepsis and those from healthy controls (Fig. 1).

Results of GO and KEGG enrichment analysis. For the upregulated DEGs, all were enriched in 51 GO BP terms and two KEGG pathways. MAP3K14 was enriched in the apoptosis pathway (Table I). Downregulated DEGs were enriched in 37 GO BP terms and five KEGG pathways, including the adipocytokine and calcium signaling pathways (Table II).

Construction of the PPI network. The PPI network was constructed using all DEGs and included 292 nodes (individual proteins) and 731 edges (interaction events), as shown in Fig. 2.
In the PPI network, MYC interacted with MAP3K14 and GTPBP4. The interacting proteins of BYSL included GTPBP4, NOP14, NOP2, NOB1, AATF and TRMT6. Following analysis of the network topology, eight DEGs (degree $>20$ ) were identified (Table III), including MYC (degree=29), NOP2 (degree=29), GTPBP4 (degree=27), BYSL (degree=25) and AATF (degree=22). The degree represents the number of interactions with other proteins.

Function of the PPI network module. The MCODE module with the highest score (14.286) in the PPI network was identified; it included 15 upregulated DEGs and 100 edges. The function of this module was enriched in ribosome biogenesis following GO analysis (Fig. 3). The top five GO terms in the module were analyzed using GO analysis, and the results showed that NOP14, NOP2, AATF, GNL2, GTPBP4, BYSL and TRMT6 were key genes in the module (Fig. 4).

Construction of the integrated DEG-miRNA-TF regulatory network. The integrated regulatory network was constructed with the target DEGs, miRNAs and TFs (Fig. 5). In the network, hsa-miR-150 was identified, which was the only miRNA associated with sepsis. Its target DEGs, including MYLK3, are shown in Table IV. A total of 21 TFs were identified in the 
Table III. Eight differentially expressed genes (degree $>20$ ) in the network topology.

\begin{tabular}{lcrr}
\hline Gene & Degree & Betweenness & Closeness \\
\hline MYC & 29.0 & $14,147.05$ & 0.054 \\
NOP2 & 29.0 & $4,228.61$ & 0.053 \\
GTPBP4 & 27.0 & $5,434.96$ & 0.053 \\
ACACB & 25.0 & $12,036.69$ & 0.053 \\
BYSL & 25.0 & $1,432.08$ & 0.052 \\
MYH6 & 23.0 & $5,115.25$ & 0.053 \\
AATF & 22.0 & $4,423.36$ & 0.053 \\
NAT10 & 22.0 & $1,173.44$ & 0.052 \\
\hline
\end{tabular}
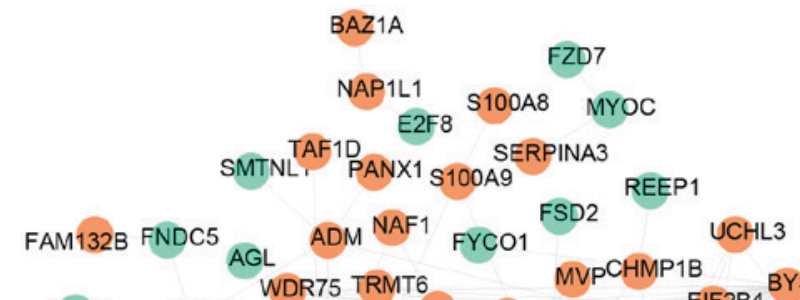

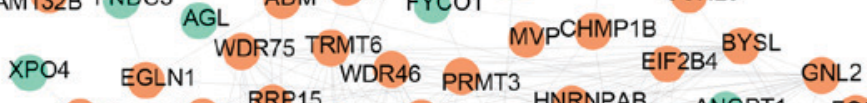

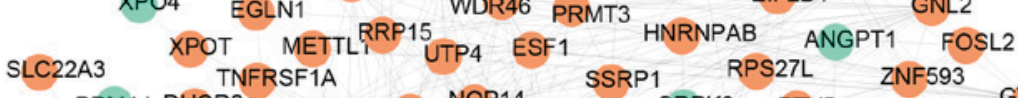
SLC22A5 PPM1J DHCR24 CMSS1DDX21 NOP14 NAT10 SRPK3 ETV5 CRFB DHX33 GTPP4 SLC47A1 GADD45A PNMA1 WDR43 RIOK1 STAM CBFB DHX33 ASB11 KLHL15 ABCB4 MIOS PUS7 DENND4ANOB1 RPL22LJUNBCCDCANFIL3 MT1M EIF3DPRMT5 KIAA0922 HEY1 MT1FNMRK2 PDP1 AATFNOP2 PLA1A MT1X TAK2 TLE1 FBXO30 HDX39A HARS CEBPDRNF20 CEBPA MN1 UGCEIK3C2EPLCD4 PLA2G2A MT1E NQ01 IRAK1 CTPS1 ETF1 PSMD\& UBFD1 KPNA2 RPN2
GSR CDCA7ICTSD

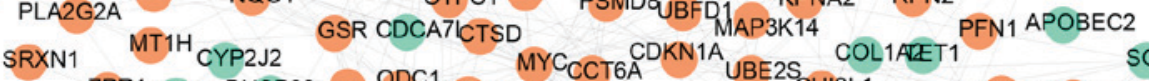

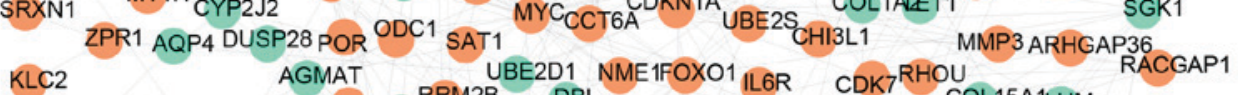

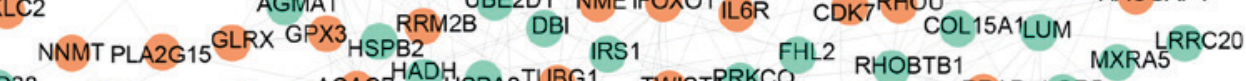

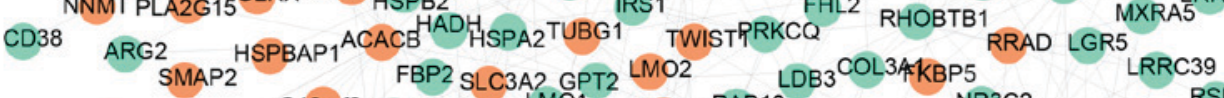

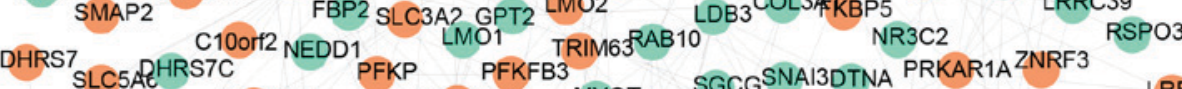

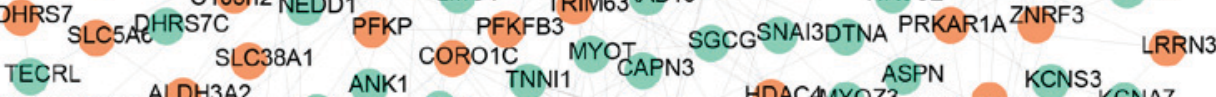

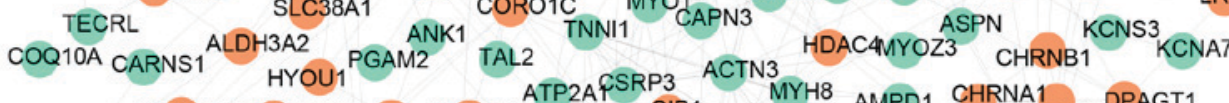
AKR1A1 CDC42EP3 ${ }_{\text {DNAJB11 }}^{\text {BLOC1S2 }}$ MUPCA CAV3 CIB1 MYH7B AMPD1 CHRNA1

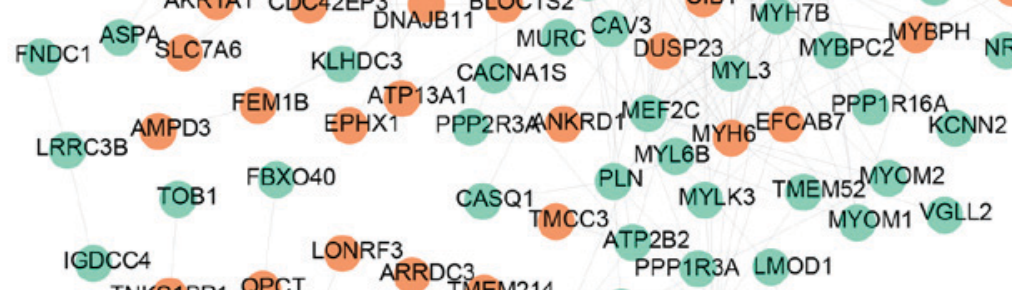
TNKS1BP1 QPCT ARRDC3 3 TMEM214 ITGB1BP2 ${ }_{\text {RNF150 ERRFI1 }}^{\text {KLHL31 }}$

Figure 2. PPI network of DEGs. All upregulated and downregulated DEGs were used to construct a PPI network. Orange nodes represent upregulated DEGs and green nodes represent downregulated DEGs. The edges between them represent PPIs. DEGs, differentially expressed genes; PPI, protein-protein interaction.

network, comprising 14 upregulated DEGs, including $M Y C$, WDR46, IPO4 and ZNF593, and seven downregulated DEGs (Table V).

\section{Discussion}

In the present study, there were 259 upregulated and 204 


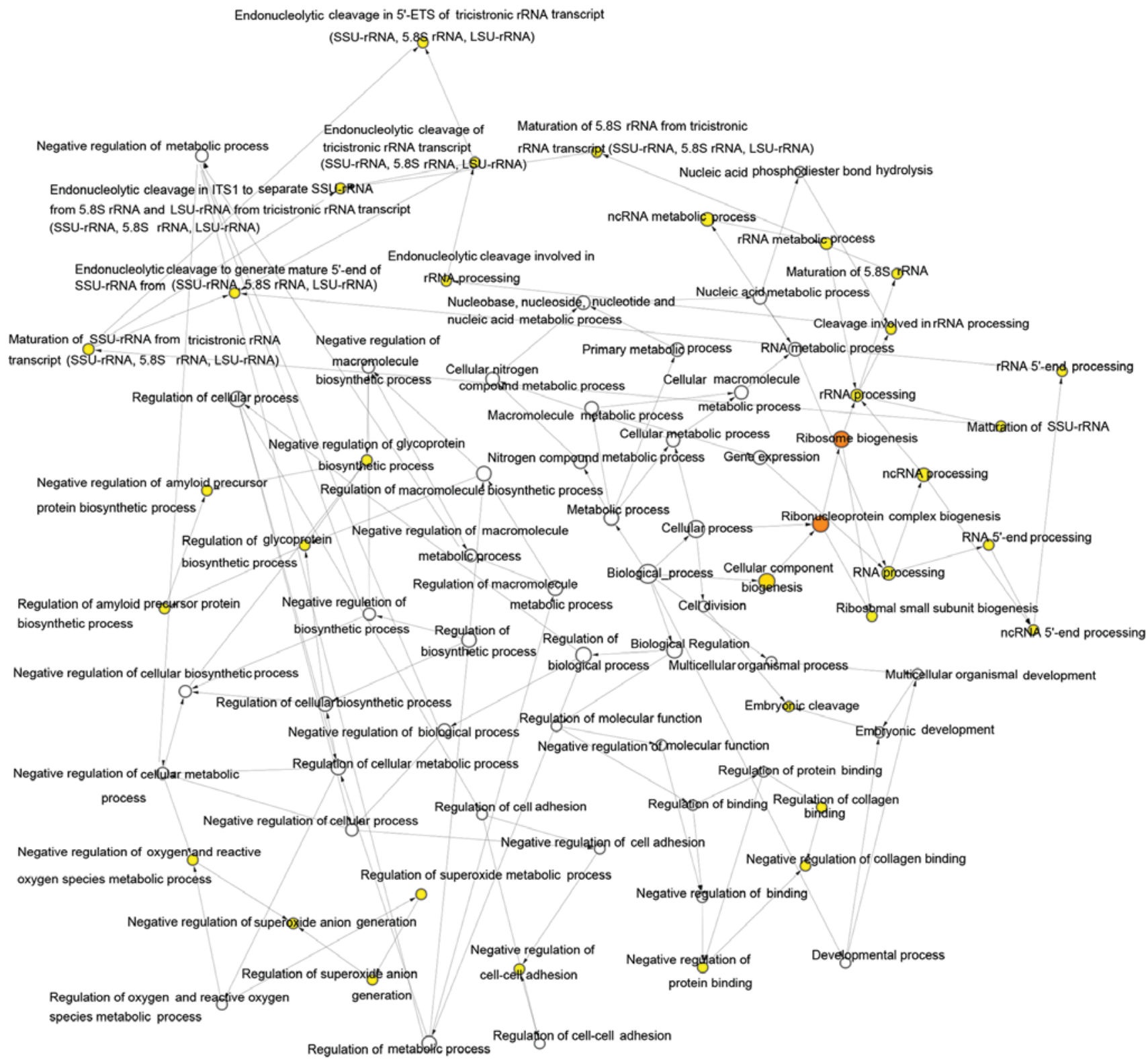

Figure 3. GO-enriched terms in the MCODE module. The module contains the highest number of genes (15 upregulated differentially expressed genes) of all modules identified via MCODE from the protein-protein interaction network and has the highest MCODE score (14.286). The yellow color indicates GO terms with a significant difference; white indicates GO terms for which the difference is not significant. Larger nodes and darker colors represent higher degrees of difference. The arrows indicate that the hierarchical structure of GO terms. GO, Gene Ontology.

downregulated DEGs in the samples from patients with sepsis, compared with those in samples from healthy controls. The upregulated DEGs were enriched in the apoptosis pathway and in the metabolism of glutathione. The downregulated DEGs were enriched in pathways, including the calcium signaling pathway and adipocytokine signaling pathway. A total of 292 nodes and 731 edges were identified in the PPI network, including eight DEGs with higher degrees (degree $>20$ ). Subsequently, key genes in the module with the maximum score $(14.286 ; 15$ upregulated DEGs and 100 edges) in the PPI network were identified. Finally, target DEGs, miRNAs and TFs were identified in samples from patients with sepsis.

In the PPI network, MYC, NOP2, GTPBP4, BYSL and AATF had a higher degree $(>20)$. The MYC protein is encoded by the MYC gene, which is associated with cell cycle, transformation and apoptosis (27). There is no direct evidence to confirm the role of $M Y C$ in sepsis. However, proinflammatory cytokines, including TNF- $\alpha$, are closely associated with sepsis characterized by SIRS (28), which may have important implications on the treatment of sepsis (29). For example, TNF- $\alpha$ protects against microvascular endothelial dysfunction in patients with sepsis by activating NF-B and p38 in endothelial cells (30). In addition, $M Y C$ promotes the expression of TNF- $\alpha$ in chronic liver diseases (31). Therefore, MYC may be crucial in the progress of sepsis by promoting the expression of TNF- $\alpha$. Qiao et al found that NOP2 and GTPBP4 may be differentially expressed in neonatal sepsis (32), which is consistent with the results of the present study. Therefore, NOP2 and GTPBP4 may be associated with the progress of sepsis. The AATF protein, also known as Che-1, is encoded by the AATF gene, which is involved in cell apoptosis (33). 


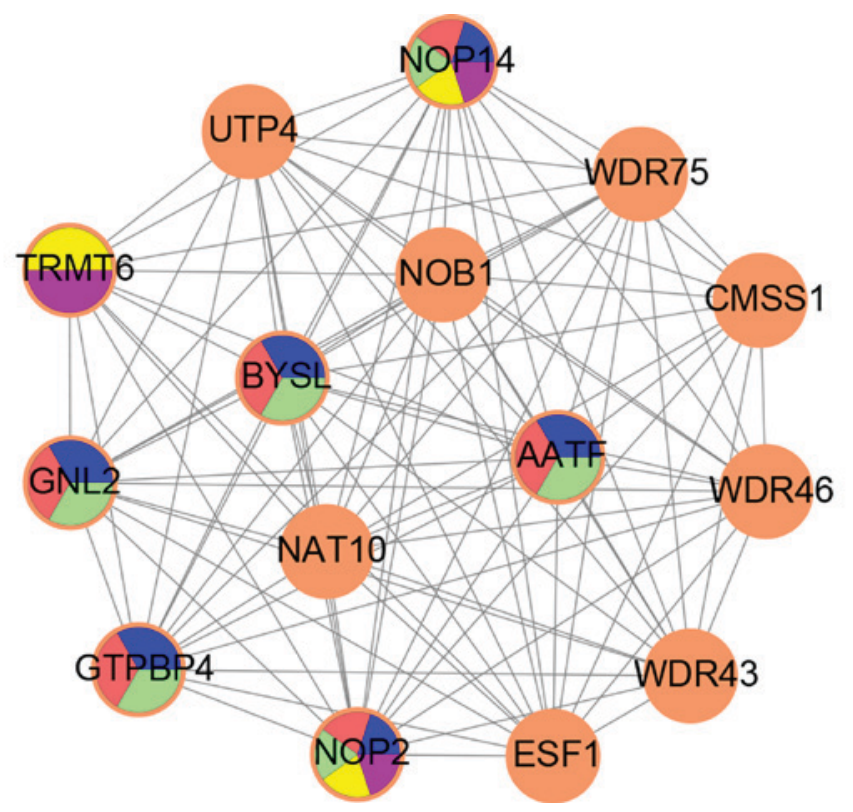

Figure 4. Genes in the top five terms in the MCODE module. Orange indicates upregulated DEGs, blue indicates biogenesis of the ribonucleoprotein complex, red indicates biogenesis of ribosomes, green indicates biogenesis of cellular components, yellow indicates ncRNA metabolic processes, purple indicates ncRNA processing. The lines indicate there are interacted with the two nodes in the network.

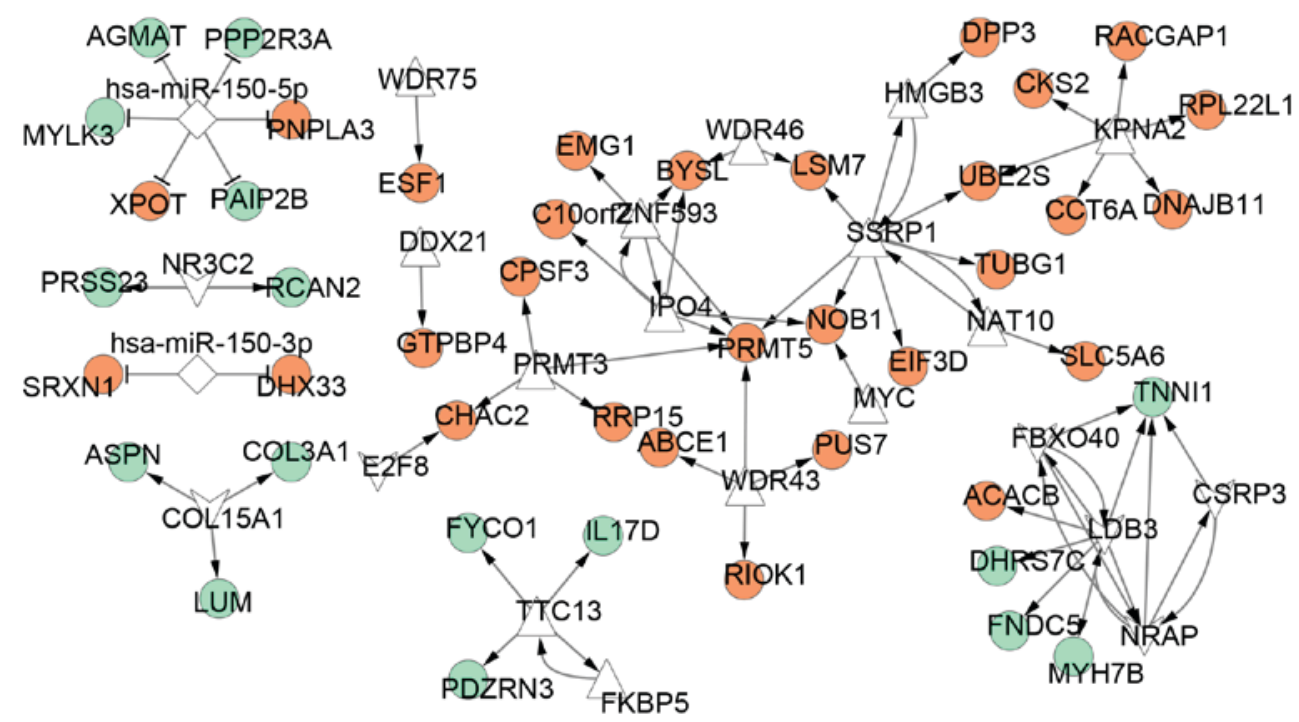

Figure 5. Integrated regulatory network. Circles represent DEGs; orange represents upregulated DEGs and green represents downregulated DEGs. The triangles represent upregulated TFs, inverted triangles represent downregulated TFs and diamonds represent microRNAs. Lines without arrows indicate regulation associations of microRNAs. The arrowed lines indicate that the regulation associations of TFs. DEGs, differentially expressed genes; TFs, transcription factors; miR, microRNA.

The overexpression of AATF interferes with MAP3K12/DLK (a protein kinase)-induced apoptosis $(34,35)$, which forms heterodimers with a leucine zipper containing TFs, including MYC, and has a regulatory role (36). There is no evidence to confirm the function of AATF in sepsis. However, AATF represents an immune- or inflammation-associated gene and encodes miRNA-2909, which is responsible for the regulation of genes involved in inflammation $(37,38)$. Therefore, AATF may be associated with inflammation in sepsis by regulating the expression of miRNA-2909.

$B Y S L$, an adhesion molecule in mammalian preimplantation embryos (39), is crucial in pre-ribosomal RNA (rRNA) processing, and its depletion leads to the accumulation of an unusual 18S rRNA precursor (40). There is no evidence to confirming its association with sepsis. In the present study, BYSL was found to closely interact with GTPBP4, NOP14, NOP2, NOB1 and AATF in the PPI network. In addition, the NOP14 gene encodes a NOP14 protein, which is involved in pre-18S rRNA processing and small ribosomal subunit assembly (41). NOB1 is involved in pre-rRNA processing and produces a mature $18 \mathrm{~S}$ rRNA in a late cytoplasmic processing step (42). Studies have reported that 16S rRNA gene amplification by real-time polymerase chain reaction and sequencing rapidly and accurately diagnose neonatal sepsis (43-45). 
Table IV. miRNA-target DEGs in the integrated DEG-miRNATF regulatory network.

miRNA Target genes

$\begin{array}{ll}\text { Hsa-miR-150-3p } & S R X N 1, D H X 33 \\ \text { Hsa-miR-150-5p } & \text { AGMAT, PPP2R3A, MYLK3, } \\ & P N P L A 3, X P O T, P A I P 2 B\end{array}$

miRNA/miR, microRNA.

Table V. TFs in the integrated DEG-miRNA-TF regulatory network.

\begin{tabular}{|c|c|}
\hline DEGs & TFs \\
\hline Upregulated & $\begin{array}{l}\text { DDX21, FKBP5, HMGB3, IPO4, KPNA2, } \\
\text { MYC, NAT10, PRMT3, SSRP1, TTC13, } \\
\text { WDR43, WDR46, WDR75, ZNF593 }\end{array}$ \\
\hline Downregulated & $\begin{array}{l}\text { COL15A1, CSRP3, E2F8, FBXO40, LDB3, } \\
\text { NR3C2, NRAP }\end{array}$ \\
\hline
\end{tabular}

DEGs, differentially expressed genes; TFs, transcription factors.

Therefore, BYSL, NOB1 and NOP14 are involved in pre-18S rRNA processing in sepsis.

In the present study, hsa-miR-150, including its target genes (MYLK3) was identified in the integrated DEG-miRNA-TF regulatory network. Zhao et al also found that the serum levels of miR-150 decreased in a rat sepsis model, providing a valuable tool in evaluating the prognosis of sepsis (11). Similarly, the expression of miR-150 was reported to be decreased in a patient with sepsis (46). A previous study found that $M L C K$, including $M Y L K 1, M Y L K 2$ and MYLK3, may not upregulate endothelial permeability induced by LPS exposure, whereas hyper-permeability was observed in sepsis and other inflammatory situations (47). TNF- $\alpha$, LPS and $18 \%$ cyclic stretch each increased the activity of a MYLK gene 3 'untranslated region luciferase reporter, and induction was reduced by mimics of each miRNA (48). As reported in the above study, a negative correlation was found between the expression of miR-150 and white blood cell counts, and the levels of IL-6, C-reactive protein and TNF- $\alpha$ (11). In the present study, MYLK3 was identified as a downregulated DEG in sepsis and one of the target genes of miR-150. These findings suggested that the expression of miR-150 is increased by upregulating $M Y L K 3$, which provides a direction for sepsis therapy by developing a novel drug targeting MYLK3 in miR-150.

There were a number of limitations in the present study, with the exception of the above finding. All predicted results require confirmation by laboratory data, however, there are currently insufficient samples from patients with sepsis. In future investigations, the expression of the DEGs discussed above requires validation using large-scale samples. In addition, future investigations aim to confirm the interactions of DEGs, regulatory associations between TFs and DEGs, and possible pathways underlying these gene alterations.

In conclusion, a total of 259 upregulated DEGs, including $M Y C, A A T F$ and BYSL, and 204 downregulated DEGs, including $M Y L K 3$, were identified from patients with sepsis. NOP14, NOP2, AATF, GTPBP4, BYSL, MYC and MYLK3 were key genes in sepsis, which may be involved in pre-18S rRNA processing in sepsis or may be associated with inflammation in sepsis by regulating the expression of miRNA-2909. In addition, miR-150 and its target genes, including MYLK3, were found to be key in sepsis. The findings may provide further understanding of the pathogenesis of sepsis and assist in developing a novel target drug for the treatment of sepsis.

\section{References}

1. Tsai D, Stewart P, Goud R, Gourley S, Hewagama S, Krishnaswamy S, Wallis SC, Lipman J and Roberts JA: Total and unbound ceftriaxone pharmacokinetics in critically ill Australian Indigenous patients with severe sepsis. Int J Antimicrob Agents 48: 748-752, 2016.

2. Fleischmann C, Scherag A, Adhikari NK, Hartog CS, Tsaganos T, Schlattmann P, Angus DC and Reinhart K; International Forum of Acute Care Trialists: Assessment of Global incidence and mortality of Hospital-treated Sepsis. Current estimates and limitations. Am J Respir Crit Care Med 193: 259-272, 2016.

3. Cheng B, Xie G, Yao S, Wu X, Guo Q, Gu M, Fang Q, Xu Q, Wang D, Jin Y, et al: Epidemiology of severe sepsis in critically ill surgical patients in ten university hospitals in China. Crit Care Med 35: 2538-2546, 2007.

4. Wallisch JS, Pang D, Carcillo JA and Aneja RK: Implementation of guidelines to treat pediatric sepsis: Cookbook medicine or the force awakens! Pediatr Crit Care Med 17: 884-885, 2016.

5. Baghel K, Srivastava RN, Chandra A, Goel SK, Agrawal J, Kazmi HR and Raj S: TNF- $\alpha$, IL- 6 , and IL- 8 cytokines and their association with TNF- $\alpha-308$ G/A polymorphism and postoperative sepsis. J Gastrointest Surg 18: 1486-1494, 2014.

6. Thimmulappa RK, Lee H, Rangasamy T, Reddy SP, Yamamoto M, Kensler TW and Biswal S: Nrf2 is a critical regulator of the innate immune response and survival during experimental sepsis. J Clin Invest 116: 984-995, 2006.

7. Coquerel D, Neviere R, Delile E, Mulder P, Marechal X, Montaigne D, Renet S, Remy-Jouet I, Gomez E, Henry JP, et al: Gene deletion of protein tyrosine phosphatase 1B protects against sepsis-induced cardiovascular dysfunction and mortality. Arterioscler Thromb Vasc Biol 34: 1032-1044, 2014.

8. Kloosterman WP, Wienholds E, de Bruijn E, Kauppinen S and Plasterk RH: In situ detection of miRNAs in animal embryos using LNA-modified oligonucleotide probes. Nat Methods 3: 27-29, 2006.

9. Cifuentes D, Xue H, Taylor DW, Patnode H, Mishima Y, Cheloufi S, Ma E, Mane S, Hannon GJ, Lawson ND, et al: A Novel miRNA processing pathway independent of dicer requires Argonaute2 catalytic activity. Science 328: 1694-1698, 2010.

10. Goodwin AJ, Guo C, Cook JA, Wolf B, Halushka PV and Fan H: Plasma levels of microRNA are altered with the development of shock in human sepsis: An observational study. Crit Care 19: 440, 2015.

11. Zhao X, Wang $\mathrm{Y}$ and Chen Z: The predict value of serum miRNA150 on sepsis prognosis. Int J Clin Exp Med 9: 18367-18372, 2016

12. Fredriksson K, Tjäder I, Keller P, Petrovic N, Ahlman B, Schéele C, Wernerman J, Timmons JA and Rooyackers O: Dysregulation of mitochondrial dynamics and the muscle transcriptome in ICU patients suffering from sepsis induced multiple organ failure. PLoS One 3: e3686, 2008.

13. Fredriksson K, Tjäder I, Keller P, Petrovic N, Ahlman B, Schéele C, Wernerman J, Timmons JA and Rooyackers O: Dysregulation of mitochondrial dynamics and the muscle transcriptome in ICU patients suffering from sepsis induced multiple organ failure. PLoS One 3: e3686, 2008. 
14. Irizarry RA, Hobbs B, Collin F, Beazer-Barclay YD, Antonellis KJ, Scherf U and Speed TP: Exploration, normalization, and summaries of high density oligonucleotide array probe level data. Biostatistics 4: 249-264, 2003.

15. Diboun I, Wernisch L, Orengo CA and Koltzenburg M: Microarray analysis after RNA amplification can detect pronounced differences in gene expression using limma. BMC Genomics 7: 252, 2006.

16. Ashburner M, Ball CA, Blake JA, Botstein D, Butler H, Cherry JM, Davis AP, Dolinski K, Dwight SS, Eppig JT, et al: Gene ontology: Tool for the unification of biology. The gene ontology consortium. Nat Genet 25: 25-29, 2000.

17. Ogata H, Goto S, Sato K, Fujibuchi W, Bono H and Kanehisa M: KEGG: Kyoto encyclopedia of genes and genomes. Nucleic Acids Res 27: 29-34, 1999.

18. Huang DW, Sherman BT, Tan Q, Collins JR, Alvord WG, Roayaei J, Stephens R, Baseler MW, Lane HC and Lempicki RA: The DAVID gene functional classification tool: A novel biological module-centric algorithm to functionally analyze large gene lists. Genome Biol 8: R183, 2007.

19. von Mering C, Huynen M, Jaeggi D, Schmidt S, Bork P and Snel B: STRING: A database of predicted functional associations between proteins. Nucleic Acids Res 31: 258-261, 2003.

20. Tang Y, Li M, Wang J, Pan Y and Wu FX: CytoNCA: A cytoscape plugin for centrality analysis and evaluation of protein interaction networks. Biosystems 127: 67-72, 2015.

21. Bader GD and Hogue CW: An automated method for finding molecular complexes in large protein interaction networks. BMC Bioinformatics 4: 2, 2003.

22. Garcia O, Saveanu C, Cline M, Fromont-Racine M, Jacquier A, Schwikowski B and Aittokallio T: GOlorize: A cytoscape plug-in for network visualization with Gene Ontology-based layout and coloring. Bioinformatics 23: 394-396, 2007.

23. Dweep H, Sticht C, Pandey P and Gretz N: miRWalk-database: Prediction of possible miRNA binding sites by 'walking' the genes of three genomes. J Biomed Inform 44: 839-847, 2011.

24. Dweep H and Gretz N: miRWalk2.0: A comprehensive atlas of microRNA-target interactions. Nat Methods 12: 697, 2015.

25. Zheng G, Tu K, Yang Q, Xiong Y, Wei C, Xie L, Zhu Y and Li Y: ITFP: An integrated platform of mammalian transcription factors. Bioinformatics 24: 2416-2417, 2008.

26. Matys V, Kel-Margoulis OV, Fricke E, Liebich I, Land S, Barre-Dirrie A, Reuter I, Chekmenev D, Krull M, Hornischer K, et al: TRANSFAC and its module TRANSCompel: Transcriptional gene regulation in eukaryotes. Nucleic Acids Res 34 (Database Issue): D108-D110, 2006.

27. Koh CM, Sabò A and Guccione E: Targeting MYC in cancer therapy: RNA processing offers new opportunities. Bioessays 38: 266-275, 2016

28. Roderburg C, Benz F, Schüller F, Pombeiro I, Hippe HJ, Frey N, Trautwein C, Luedde T, Koch A, Tacke F and Luedde M: Serum levels of TNF receptor ligands are dysregulated in sepsis and predict mortality in critically Ill patients. PLoS One 11: e0153765, 2016.

29. Hotchkiss RS, Monneret G and Payen D: Immunosuppression in sepsis: A novel understanding of the disorder and a new therapeutic approach. Lancet Infect Dis 13: 260-268, 2013.

30. Liang Y, Li X, Zhang X, Li Z, Wang L, Sun Y, Liu Z and Ma X: Elevated levels of plasma TNF- $\alpha$ are associated with microvascular endothelial dysfunction in patients with sepsis through activating the NF- $\mathrm{KB}$ and $\mathrm{p} 38$ mitogen-activated protein kinase in endothelial cells. Shock 41: 275-281, 2014

31. Liu T, Zhou Y, Ko KS and Yang H: Interactions between Myc and mediators of inflammation in chronic liver diseases. Mediators Inflamm 2015: 276850, 2015.

32. Qiao X, Zhu S, Zhang S and Dong H: Disrupted pathways associated with neonatal sepsis: Combination of protein-protein interactions and pathway data. BioChip J 11: 1-7, 2017.
33. Liu M, Wang D and Li N: Che-1 gene silencing induces osteosarcoma cell apoptosis by inhibiting mutant p53 expression. Biochem Biophys Res Commun 473: 168-173, 2016.

34. Smigiel R, Marcelis C, Patkowski D, de Leeuw N, Bednarczyk D, Barg E, Mascianica K, Maria Sasiadek M and Brunner H: Oesophageal atresia with tracheoesophageal fistula and anal atresia in a patient with a de novo microduplication in 17q12. Eur J Med Genet 57: 40-43, 2014.

35. De Nicola F, Bruno T, Iezzi S, Di Padova M, Floridi A, Passananti C, Del Sal G and Fanciulli M: The prolyl isomerase Pin1 affects Che-1 stability in response to apoptotic DNA damage. J Biol Chem 282: 19685-19691, 2007.

36. Baker ST, Opperman KJ, Tulgren ED, Turgeon SM, Bienvenut W and Grill B: RPM-1 uses both ubiquitin ligase and phosphatase-based mechanisms to regulate DLK-1 during neuronal development. PLoS Genet 10: e1004297, 2014.

37. Sharma S, Singh D and Kaul D: AATF RNome has the potential to define post mortem interval. Forensic Sci Int 247: e21-e24, 2015.

38. Lee SJ, Lee EJ, Kim SK, Jeong P, Cho YH, Yun SJ, Kim S, Kim GY, Choi YH, Cha EJ, et al: Identification of pro-Inflammatory cytokines associated with muscle invasive bladder cancer; the roles of IL-5, IL-20, and IL-28A. PLoS One 7: e40267, 2012.

39. Hashimoto M, Sato T, Muroyama Y, Fujimura L, Hatano M and Saito T: Nepro is localized in the nucleolus and essential for preimplantation development in mice. Dev Growth Differ 57: 529-538, 2015.

40. Carron C, O'Donohue MF, Choesmel V, Faubladier M and Gleizes PE: Analysis of two human pre-ribosomal factors, bystin and hTsrl, highlights differences in evolution of ribosome biogenesis between yeast and mammals. Nucleic Acids Res 39: 280-291, 2011.

41. Liu PC and Thiele DJ: Novel stress-responsive genes EMG1 and NOP14 encode conserved, interacting proteins required for $40 \mathrm{~S}$ ribosome biogenesis. Mol Biol Cell 12: 3644-3657, 2001

42. Zhang J, McCann KL, Chen Q, Gonzalez LE, Baserga SJ and Hall TM: Nop9 is a PUF-like protein that prevents premature cleavage to correctly process pre-18S rRNA. Nat Commun 7: 13085, 2016.

43. El Gawhary S, El-Anany M, Hassan R, Ali D and El Gameel el Q: The role of 16S rRNA gene sequencing in confirmation of suspected neonatal sepsis. J Trop Pediatr 62: 75-80, 2016.

44. Mithal LB, Malczynski M, Green SJ, Qi C, Yogev R and Mestan K: Deep sequencing of 16S rRNA gene amplicons to screen umbilical cord blood of preterm infants. Open Forum Infectious Dis 3: 2234, 2016.

45. Midan DA, Abo El Fotoh WMM and El Shalakany AH: The potential role of incorporating real-time PCR and DNA sequencing for amplification and detection of 16S rRNA gene signatures in neonatal sepsis. J Matern Fetal Neonatal Med 30: 1476-1483, 2017.

46. Ticlea M, Bratu LM, Bodog F, Bedreag OH, Rogobete AF and Crainiceanu ZP: The use of exosomes as biomarkers for evaluating and monitoring critically Ill polytrauma patients with sepsis. Biochem Genet 55: 1-9, 2017.

47. Liu H, Yu X, Yu S and Kou J: Molecular mechanisms in lipopolysaccharide-induced pulmonary endothelial barrier dysfunction. Int Immunopharmacol 29: 937-946, 2015.

48. Adyshev DM, Moldobaeva N, Mapes B, Elangovan V and Garcia JG: MicroRNA regulation of nonmuscle myosin light chain kinase expression in human lung endothelium. Am J Respir Cell Mol Biol 49: 58-66, 2013.

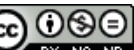

This work is licensed under a Creative Commons Attribution-NonCommercial-NoDerivatives 4.0 International (CC BY-NC-ND 4.0) License. 